\title{
Registro chileno de técnicas de reproducción asistida, 2011
}

\author{
Juan E. Schwarze M. ${ }^{1}$, Javier A. Crosby R. ${ }^{2}$, David Vantman B. ${ }^{3}$, Patricio Masoli C. ${ }^{4}$, Italo \\ Ciuffardi C. ${ }^{5}$, Pablo Céspedes P. ${ }^{6}$, Patricio González S. ${ }^{7}$, Gonzalo Duque A. ${ }^{8}$, Ricardo \\ Pommer T. ${ }^{9}$
}

${ }^{1}$ Presidente Sociedad Chilena de Medicina Reproductiva. ${ }^{2}$ Secretario Sociedad Chilena de Medicina Reproductiva.

${ }^{3}$ Centro de Estudios Reproductivos. ${ }^{4}$ Clínica de la Mujer y Medicina Reproductiva. ${ }^{5}$ Clínica Sanatorio Alemán. ${ }^{6}$ Instituto de Investigación Materno Infantil de la Universidad de Chile. ${ }^{7}$ Unidad de Medicina Reproductiva Clínica Alemana.

${ }^{8}$ Unidad de Medicina Reproductiva Clínica Las Condes. ${ }^{9}$ Unidad de Medicina Reproductiva Clínica Monteblanco. Chile.

\section{RESUMEN}

Presentamos el Registro Chileno de Técnicas de Reproducción Asistida realizadas en el año 2011. Siete centros reportaron un total de 1.918 ciclos de reproducción asistida: 161 ciclos de fecundación in vitro (FIV), 1.266 ciclos de inyección intracitoplasmática de espermatozoides (ICSI), 325 ciclos de transferencia de embriones criopreservados, y 166 ciclos de transferencia de embriones producto de ovodonación. La tasa de parto por aspiración en ciclos de FIV fue 18,6\% y en ciclos de ICSI fue 22,0\%. La tasa de parto por transferencia embrionaria en ciclos de transferencia de embriones criopreservados fue $21,5 \%$; en ciclos de transferencia de embriones producto de ovodonación fue 41,9\%. La edad promedio de las mujeres sometidas a ciclos de IVF/ICSI fue 35,7 años (rango: $22-48$ años). El $38 \%$ de los ciclos iniciados fue en mujeres $\leq 34$ años. A diferencia de años anteriores, la media de embriones transferidos no presentó una disminución, estabilizándose en 2,1. La frecuencia global de parto múltiple en IVF/ICSI fue 24\%; $3 \%$ gemelar y $1 \%$ parto triple y mayor. Concluimos que la tasa de parto de los ciclos de IVF/ICSI se mantiene relativamente estable en relación a años anteriores. Gracias a la criopreservación y la transferencia secuencial de toda la cohorte de embriones producidos, la frecuencia de partos triple descendió considerablemente. Sin embargo, la alta frecuencia de parto doble no ha experimentado ninguna disminución y debe ser el próximo desafío.

\section{PALABRAS CLAVES: Chile, registro de reproducción asistida}

\section{SUMMARY}

We present the Chilean Registry of Assisted Reproductive Technologies performed in 2011. For the first time this registry is performed on a case-by-case basis. Seven centres reported 1,918 cycles: 161 in vitro fertilisation cases (IVF), 1,366 intracytoplasmatic sperm injection cases (ICSI), 325 cryiopreserved embryo transfers, and 158 oocyte donation cycles. The delivery rate per oocyte pick up for IVF and ICSI cycles was $18.6 \%$ and $22.0 \%$, respectively. The delivery rate per embryo transfers for cryopreserved embryo transfer and oocyte donation were $21.5 \%$ and $41.9 \%$, respectively. The mean age of woman undergoing IVF/ICSI was 35.7 years ( $\min 22 \max 48$ years); $38 \%$ of initiated cycles were performed in women aged $\leq 34$ years. As in previous years, the mean number of embryos transferred declined slightly, reaching 2.1. The multiple delivery rate was $24 \%: 23 \%$ twin and $1 \%$ triplet and higher. In conclusion the delivery rate in IVF/ICSI 
cycles remains stable. Thanks to cryopreservation and posterior embryo transfer, the rate of triplet-delivery continues to diminish. However, the rate of twin delivery has not diminished, and remains a challenge.

\section{KEY WORDS: Chile, IVF registry}

\section{INTRODUCCIÓN}

El presente reporte corresponde al Registro Chileno de Reproducción Asistida, con información referente a los ciclos de reproducción asistida comenzados entre el 1 de Enero y el 31 de Diciembre del 2011. Esta es la segunda vez que esta información es publicada en una revista científica para facilitar el acceso tanto a especialistas como a pacientes. El reporte anterior fue publicado en esta misma revista. Asimismo, en la página web de la Sociedad Chilena de Medicina Reproductiva (www. socmer.cl) se puede encontrar los datos correspondientes a los años 1990-2009.

\section{MATERIAL Y MÉTODOS}

Estos datos corresponden a la información entregada por los centros chilenos de medicina reproductiva al Registro Latinoamericano de Reproducción Asistida (REDLARA).

Recolección de datos. Los ciclos de reproducción asistida considerados incluyen ciclos de fecundación in vitro (FIV), inyección intracitoplasmática de espermatozoides (ICSI), transferencia a la trompa de gametos y zygotos (GIFT/TOMI), transferencia de embriones producto de ovodonación (OD), transferencia de embriones congelados/descongelados (TEC), y ciclos con diagnóstico genético preimplantacional (PGD). Adicionalmente, se incluye ciclos de inseminación intrauterina utilizando semen del marido (IAM) o de donante (IAD).

Este reporte incluye datos de ciclos realizados entre el 1 de Enero y el 31 de Diciembre del 2011, y los niños nacidos de estos procedimientos. Los datos obtenidos son entregados en forma voluntaria por los centros chilenos miembros de la REDLARA. Cada centro miembro tiene una clave individual con la que puede acceder al programa en línea disponible a través del sitio web de REDLARA; esto le permite incluir la información en el registro, a medida que se desarrolla el caso. Una alternativa, si el centro así lo prefiere, puede completar planillas ad hoc, y subir la información al servidor del registro. La información proporcionada por los centros es periódicamente confirmada por el Comité de Acreditación de REDLARA.
La oficina central del Registro Latinoamericano tiene acceso inmediato a la información y puede verificar la consistencia en la información entregada. Así, en conjunto con el programa de acreditación de REDLARA, se puede certificar la veracidad y consistencia en los datos reportados por cada centro.

Validación de los datos. Los resultados de los tratamientos realizados son proporcionados por los centros y validados por un programa computacional. Si existe alguna inconsistencia entre diferentes partes de la información, ésta es reportada al centro y se inicia un proceso de interacción entre la oficina del registro y el centro hasta aclarar las inconsistencias.

Limitaciones de los datos. Cada caso representa un ciclo de tratamiento y no a una paciente. De este modo, es posible que una mujer pueda ser sometida a más de un tratamiento dentro de un año, y tener más de un embarazo. Estimamos que los datos faltantes no superan el $5 \%$ de la información. Esto es especialmente cierto en el caso de la información perinatal, ya que muchos centros miembros no están asociados a servicios de obstetricia lo que a veces dificulta tener la información acerca de los partos y neonatos generados por las técnicas de reproducción asistida. No todos los centros de medicina reproductiva de Chile están acreditados o asociados a REDLARA, de modo que este reporte no incluye todos los casos realizados en Chile. Sin embargo, estimamos que la mayoría de los procedimientos realizados en el país son reportados a través del RLA.

Análisis estadístico. En el caso de variables categóricas se comprobó independencia de variables mediante el test de Chi cuadrado. En el caso de análisis multivariado, se utilizó regresión logística y consideró que las variables se asociaban significativamente cuando el intervalo de confianza del OR no pasaba por uno. Se consideró un error alpha de $5 \%$ como el nivel de significancia estadística.

\section{RESULTADOS}

Participación: Siete centros registraron los ciclos de procedimientos de reproducción asistida realizados en el año 2011, los mismos centros que reportaron en el año 2010. 
Método de reporte: El promedio de ciclos registrados anualmente por los centros, esto es la suma de los ciclos iniciados de FIV, ICSI y GIFT/TOMI, más TEC (propios y producto de OD), y la transferencia de embriones producto de OD, fue de 287 (236 en el 2010; rango: 95-533).

Número de ciclos por técnica y acceso: El número total de procedimientos de reproducción asistida registrados fue de 1.918 (Tabla I). Esto representa un aumento del $16 \%$ en relación al 2010 , cuando se registraron 1.650 ciclos de técnicas de reproducción asistida. De los 1.427 ciclos autólogos iniciados, $161(11 \%)$ correspondieron a ciclos iniciados de FIV y 1.266 (89\%) a ciclos iniciados de ICSI. Esto no varió importantemente en relación al año 2010 , cuando los ciclos de FIV y de ICSI representaban el $12 \%$ y el $88 \%$, respectivamente. Seis clínicas reportaron en total 325 TEC ( 255 ciclos en el 2010); y cinco clínicas reportaron 166 (158 ciclos en el 2010) ciclos de transferencia de embriones producto de OD (129 frescos y 37 TEC). En el
$72 \%$ de las transferencias de embriones frescos, el origen de los ovocitos fue de donantes que fueron sometidas a hiperestimulación ovárica controlada y culdocentesis, con el fin de donar la totalidad de los ovocitos recuperados (53\% en el 2010). La Tabla I también muestra el acceso a técnicas de reproducción asistida, que llegó a 223 ciclos por millón de mujeres en edad fértil.

Embarazos y partos: La Tabla II muestra las tasas de embarazo y parto por aspiración folicular en ciclos de ICSI y FIV. En el caso de ICSI, la tasa de embarazo clínico por aspiración fue 32,5\% (21,4\% en el 2010$)$, mientras que la tasa de parto por aspiración fue $22 \%$ (21,3\% en el 2010$)$. En el caso de ciclos de FIV, estos valores fueron inferiores: la tasa de embarazo clínico por aspiración fue $29,2 \%(27,1 \%$ en el 2010$)$, y la tasa de parto por aspiración fue $18,6 \%$ (26,2\% en el 2010). La Tabla III muestra las tasas de embarazo clínico y parto por transferencia embrionaria en ciclos de TEC, OD y OD(TEC).

\section{Tabla I \\ CICLOS DE REPRODUCCIÓN ASISTIDA REGISTRADOS POR CENTROS CHILENOS A LA REDLARA, 2011}

\begin{tabular}{ccccccc}
\hline Centros & \multicolumn{4}{c}{ Ciclos de reproducción asistida } & & Acceso \\
& FIV & ICSI & TEC & OD * & Total & $\begin{array}{c}(1.000 .000 \text { mujeres } \\
15-45 \text { años })\end{array}$ \\
\hline 7 & 161 & 1.266 & 325 & 166 & 1.918 & 233,0 \\
\hline
\end{tabular}

* Transferencias de embriones frescos y congelados

Tabla II

TASAS DE EMBARAZO Y PARTO POR ASPIRACIÓN, ICSI/FIV, CHILE 2011

\begin{tabular}{|c|c|c|c|c|c|}
\hline Asp & $\begin{array}{c}\text { ICSI } \\
\text { EC/asp }\end{array}$ & Parto/asp & Asp & $\begin{array}{c}\text { FIV } \\
\text { EC/asp }\end{array}$ & Parto/asp \\
\hline 1.266 & $32,5 \%$ & $21,5 \%$ & 161 & $29,2 \%$ & $18,6 \%$ \\
\hline
\end{tabular}

Asp: aspiraciones; EC/asp: tasa de embarazo clínico por aspiración (\%); Parto/asp: tasa de parto por aspiraciones (\%)

Tabla III

TASA DE EMBARAZOY PARTO POR TRANSFERENCIA EMBRIONARIA EN CICLOS DE TECY OD, CHILE 2011

\begin{tabular}{|c|c|c|c|c|c|c|c|c|}
\hline \multicolumn{3}{|c|}{ TEC } & \multicolumn{3}{|c|}{ OD (fresco) } & \multicolumn{3}{|c|}{ OD (tec) } \\
\hline $\mathrm{TE}$ & $\mathrm{EC} / \mathrm{TE}$ & Parto/TE & TE & $\mathrm{EC} / \mathrm{TE}$ & Parto/TE & TE & $\mathrm{EC} / \mathrm{TE}$ & Parto/TE \\
\hline 325 & $34,5 \%$ & $21,5 \%$ & 129 & $56,6 \%$ & $41,9 \%$ & 37 & $21,6 \%$ & $13,5 \%$ \\
\hline
\end{tabular}

TE: transferencias. EC/TE: tasa de embarazo clínico por transferencia embrionaria (\%). Parto/TE: tasa de parto por transferencia embrionaria (\%) 
En el caso de ciclos de TEC, la tasa de embarazo clínico por transferencia embrionaria fue $34,5 \%$ (27,5\% en el 2010), y de parto por transferencia embrionaria fue de $21,5 \%$ (19,2\% en el 2010). En ciclos de OD, la tasa de embarazo por transferencia embrionaria fue $56,6 \%$ ( $41,2 \%$ en el 2010$)$, y la tasa de parto por transferencia embrionaria fue $41,9 \%$ (35,3\% en el 2010$)$.

Distribución etaria: La edad promedio de las pacientes sometidas a ciclos de FIV/ICSI fue de 35,7 años (35,5 años en el 2010) (rango: 22-48 años). El $38 \%$ de los ciclos iniciados fue en mujeres $\leq 34$ años, el $41 \%$ en mujeres de 35 a 39 años, el $17 \%$ en mujeres de 40 a 42 años; y el $4 \%$ en mujeres $\geq 43$ años (Figura 1). La edad promedio de las pacientes sometidas a ciclos de TEC fue de 35,3 años (rango: 22-49 años). En el caso de ciclos de OD, la edad promedio de las donantes fue de 28 años (rango: 19-38 años) y la de las receptoras fue de 41 años (rango: 29-52 años). En los ciclos de IVF/ICSI la tasa de parto por transferencia embrionaria varió sustancialmente con la edad de la mujer. Es así como al analizar la tasa de parto por transferencia embrionaria en las siguientes categorías etarias:

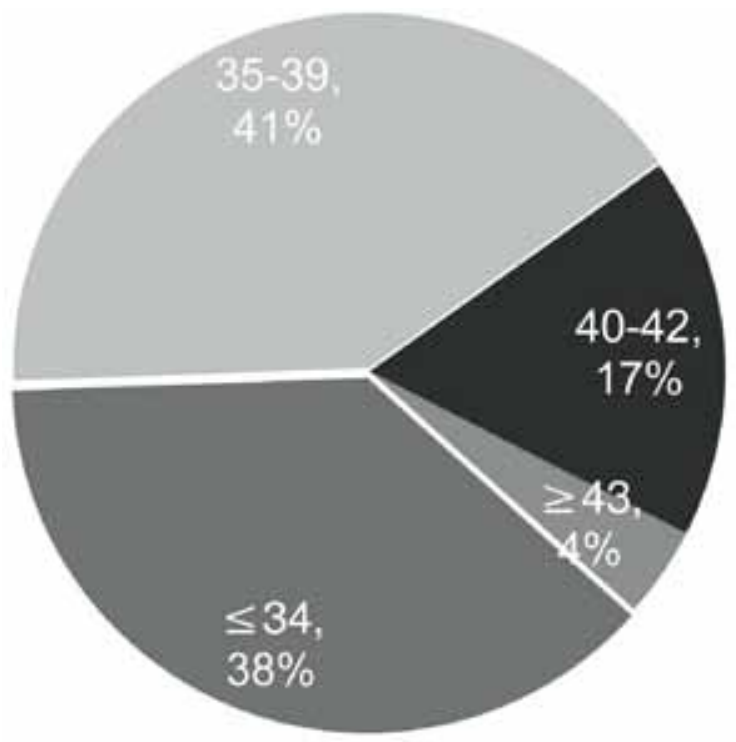

Figura 1. Distribución etaria de las mujeres sometidas a ciclos de IVF/ICSI. Chile 2011. $\leq 34$ años, 35 a 39 años, 40 a 42 años $y \geq 43$ años, la tasa de parto por ciclo iniciado disminuyó sostenidamente y ostensiblemente de $27 \%$ a $2 \%$ (Figura 2) $(p<0,001)$. La mayor edad en que se registró parto usando ovocitos propios fue 43 años, edad en la que se registró 1 caso. En los ciclos de OD, no se vio una clara relación entra la edad de la receptora y la tasa de parto. Es así como el grupo de receptoras de $\leq 34$ años tuvo una tasa de parto por transferencia de $70,0 \%$, el grupo de 35 a 39 años de $27,3 \%$, el grupo de 40 a 42 años de $44.8 \%$, y el grupo de $\geq 43$ años de $28,6 \%(p=0,005)$. No presentamos las tasas en caso de transferencia embrionaria de embriones descongelados de acuerdo a edad de la mujer, ya que se registra la edad de la mujer al momento de la transferencia embrionaria, no al momento de la criopreservación.

Número de embriones transferidos y parto múltiple: Como se muestra en la Tabla IV, se registraron en total 1.145 transferencias embrionarias en ciclos de IVF/ICSI. La media de embriones transferidos fue de 2,1; la misma que en el año $2010(2,1)$. En la mayoría de las transferencias embrionarias se transfirieron 2 embriones (72\%) (65\% en el 2010$)$, y la transferencia embrionaria de $\geq 4$ embriones representó el $3 \%$ ( $2 \%$ en el 2010 ) de los casos. En la mayoría de los ciclos, la transferencia se realizó en estadio de embrión $(n=1.028)$. La frecuencia global de parto múltiple fue $24 \%$ (22,0\% en el 2010$), 23 \%$ gemelar (21\% en el 2010), y $1 \%$ embarazo triple y mayor $(0,4 \%$ en el 2009). Asimismo, se registraron 129 transferencias embrionarias de embriones producto de OD. La media de embriones transferidos fue de 2,1 (2,0 en el 2010). El $81 \%$ de las transferencias fue de 2 embriones, y en el $14 \%$ de los casos se transfirió 3 y $\geq 4$ embriones. En la mayoría de los ciclos, la transferencia se realizó en estadio de embrión $(n=124)$. La frecuencia de parto múltiple llegó al $30 \%$, con solamente partos gemelares, no se registró ningún parto triple o mayor. En el año 2010 , la frecuencia de parto gemelar fue $28 \%$, sin partos triples o mayores. Finalmente, se registraron 325 ciclos de TEC. La media de embriones transferidos fue 1,8 y en la mayoría de los casos se transfirieron dos embriones (70\%). A diferencia de los casos anteriores, la mayoría de las transferencias se realizaron en estadio de blastocisto $(n=202)$. La frecuencia de parto múltiple fue del $23 \%$, un $19 \%$ de parto doble y un $4 \%$ de parto triple. 


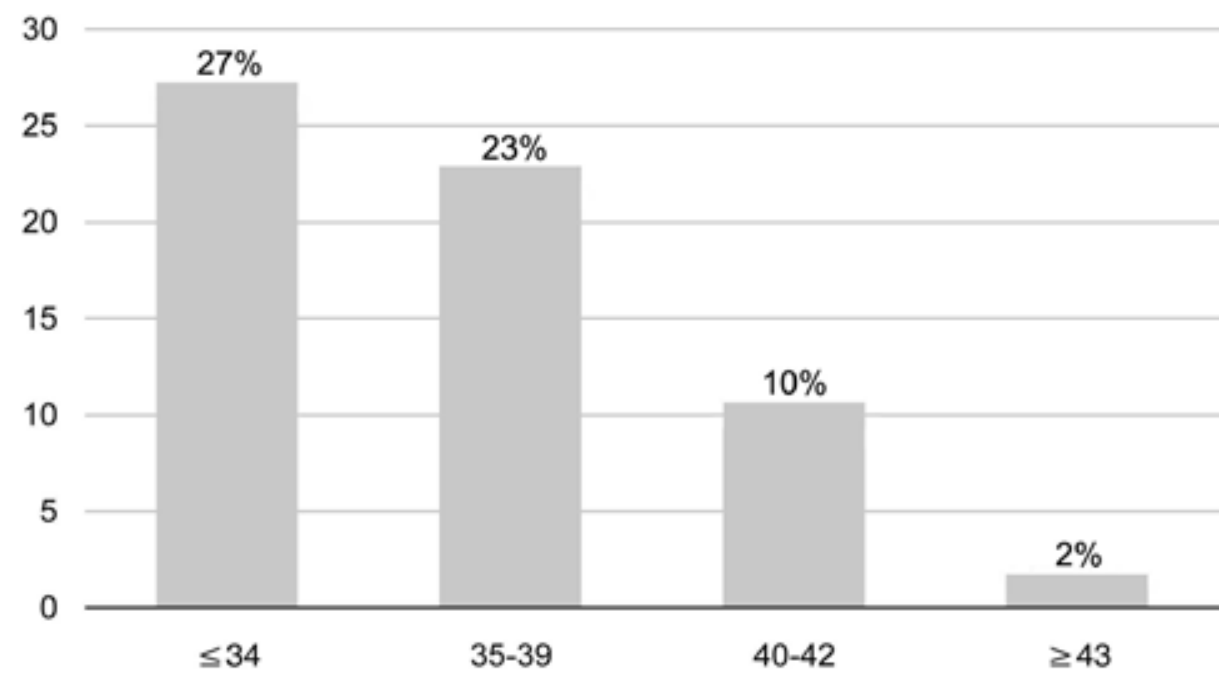

Figura 2. Tasa de parto por transferencia embrionaria en ciclos IVF/ICSI. Chile 2011.

Tabla IV

NÚMERO DE EMBRIONES TRANSFERIDOS Y ORDEN GESTACIONAL, CHILE 2011

\begin{tabular}{ccccccccc}
\hline Técnica & TE & \multicolumn{3}{c}{ Número de embriones transferidos } & \multicolumn{3}{c}{ Orden gestacional } \\
& & 1 & 2 & 3 & $\geq 4$ & Único & Doble & $\geq$ Triple \\
\hline IVF/ICSI & 1.145 & $12 \%$ & $72 \%$ & $13 \%$ & $3 \%$ & $76 \%$ & $23 \%$ & $1 \%$ \\
OD & 129 & $5 \%$ & $81 \%$ & $14 \%$ & $0 \%$ & $72 \%$ & $28 \%$ & $0 \%$ \\
TEC & 325 & $23 \%$ & $70 \%$ & $7 \%$ & $0 \%$ & $77 \%$ & $19 \%$ & $4 \%$ \\
\hline
\end{tabular}

TE: transferencias embrionarias

Transferencia electiva de uno y dos embriones: La transferencia electiva de un embrión representó el $3 \%(n=34)$ del total de transferencias, mientras que la transferencia electiva de dos embriones representó al $29 \%$ de las transferencias embrionarias $(n=330)$. Estos valores son similares a los del 2010 , cuando la transferencia electiva de un embrión representó el $4 \%(n=36)$ de las transferencias, y la transferencia electiva de dos embriones representó el 33\% $(n=330)$ de las transferencias embrionarias. En el caso de eSET, la mayoría de las transferencias fue en estadio de embrión $(n=26)$, al igual en el caso de eDET $(n=338)$. La tasa de parto por transferencia embrionaria al transferir electivamente un embrión alcanzó al 8,8\%, y al transferir electivamente dos embriones alcanzó al 35,1\%. En el grupo de mujeres $\leq 34$ años, la tasa de parto con
eSET fue mejor $(27,3 \%)$, mientras que la tasa de parto con eDET no aumentó en forma significativa $(37,6 \%)$.

Riesgos perinatales: Se registró las semanas de amenorrea al momento del parto en 436 casos. Al igual que en registros anteriores, la edad gestacional al momento del parto disminuyó con el orden gestacional. La edad gestacional al momento del parto de los partos únicos fue 37,7 semanas, de los partos dobles de 35,3 semanas y de los partos triples de 31,6 semanas. Asimismo, la frecuencia de parto pretérmino (<37 semanas) y parto pretérmino extremos (<32 semanas) fue en partos únicos 13\% y $3 \%$ respectivamente, en partos dobles $69 \%$ y $10 \%$, respectivamente, y en partos triples $100 \%$ y $71 \%$, respectivamente. Se registraron cuatro casos de muerte perinatal, en embarazos únicos con prematuridad 
extrema. Se reportaron cinco casos de síndrome de hiperestimulación ovárica y un caso de hemorragia, lo que corresponde a un riesgo de $0,5 \%$ de complicación mayor.

Diagnóstico genético preimplantacional (PGD/ $P G S)$ : Un centro reportó 42 ciclos de transferencia de embriones sometidos a PGD/PGS, ocurriendo transferencia de al menos un embrión en 16 casos. En la mitad de los casos la transferencia se realizó en estadio de blastocisto, la otra mitad en estadio de embrión. La edad promedio de las mujeres con estudio genético preimplantacional fue 40 años (rango: 28-46 años). En promedio se realizó estudio genético en cinco embriones, dos de los que fue informados como normal. Se produjeron 5 embarazos, no hubo abortos, por lo que nacieron cinco bebés únicos.

Tasa de parto acumulada: En base a la información registrada se puede estimar la tasa acumulada de parto por ciclo iniciado de FIV/ICSI. Esto corresponde a la suma de los partos obtenidos tras la transferencia de embriones descongelados/congelados y de los partos obtenidos tras la transferencia de embriones frescos. Si bien esto no corresponde a una tasa acumulada real de la pareja, es un indicador que permite simular el efecto de la transferencia de una cohorte completa de embriones. La tasa de parto acumulado por aspiración llegó al $26,5 \%$ (25,4\% en el 2010$)$ (Tabla V).

\section{DISCUSIÓN}

Presentamos el segundo registro de técnicas de reproducción asistida reportados por los centros chilenos acreditados a REDLARA. Dos son las principales fortalezas de este registro. Primero, se trata de un registro que junta los datos en forma individual y como resumen de casos. Esto permite simplificar las bases de datos y otorgar información más precisa acerca de las características de los ciclos de reproducción asistida realizados en la región. La otra ventaja es la uniformidad de la terminología utilizada por los distintos centros miembros de la RED. En efecto, todos los centros ocupan el glosario definido en el año 2006 por el Comité Internacional para Monitorizar Técnicas de Reproducción Asistida (ICMART, por sus siglas en inglés) (1).

En este año, los mismo siete centros que reportaron en el 2010, reportaron 1.918 ciclos de reproducción asistida realizados en 2011 . Esto representa un aumento del $16 \%$ en relación al registro del 2010. A pesar de esto, el acceso a las técnicas de reproducción asistida en Chile es menor que otros países latinoamericanos. Por ejemplo, en Argentina el acceso fue de 465 ciclos por millón de mujeres en edad fértil, y mucho menor que en países europeos, donde el acceso a tratamientos de reproducción asistida varía entre valores sobre diez mil por millón de mujeres en edad fértil, a poco más de quinientos ciclos por millón de mujeres en edad fértil (2).

La proporción de ciclos de ICSI sobre FIV convencional se mantiene en $89 \%$, lo mismo que en el 2010 (88\%), al igual que en años anteriores: 2009 (89\%) y 2008 (88\%). Esto a pesar que la proporción de alteraciones severas del semen es mucho menor. Esta tendencia que también se ve en el resto de Latino América, refleja probablemente los deseos de asegurar la fecundación, frente al temor de una falla de fecundación por IVF no prevista.

La tasa de parto de IVF/ICSI por ciclo aspirado llegó a $22 \%$ (21,6\% en el 2010), mientras que la tasa de parto acumulado llegó a $26,5 \%(25,4 \%$ en el 2010). Los valores registrados por centros chilenos, son superiores a lo publicado por países europeos, donde la tasa de parto por aspiración llega a $21 \%$ en ciclos de IVF, y a $20 \%$ en ciclos de ICSI. La tasa de parto acumulada llega a $22 \%$ (2).

La media de embriones transferidos en ciclos de IVF/ICSI se mantuvo similar al registro anterior $(1,2)$. Además, se registró un importante aumento en la frecuencia de transferencias electivas de uno y dos embriones. Asociado a esto, hubo un importante aumento en la frecuencia de TEC. A pesar de estas noticias tan auspiciosas, no deja de preocupar que en el $13 \%$ de los casos se transfiere tres embriones, e incluso el $3 \%$ de las transferencias corresponde a

Tabla V

TASA DE PARTO ACUMULADA CICLOS IVF/ICSI, CHILE 2011

\begin{tabular}{|c|c|c|c|}
\hline Total aspiraciones & Partos IVF/ICSI & Partos tras TEC & $\begin{array}{c}\text { Tasa de parto } \\
\text { acumulada }\end{array}$ \\
\hline 1.427 & 308 & 70 & $26,5 \%$ \\
\hline
\end{tabular}


Tabla VI

RESUMEN DE LA EXPERIENCIA, CHILE 2011

\begin{tabular}{|c|c|c|c|c|c|}
\hline & IVF/ICSI & OD & TEC & OD/TEC & Total \\
\hline Ciclos iniciados & 1.427 & 145 & 350 & 40 & 1.962 \\
\hline Transferencias embrionarias & 1.146 & 129 & 325 & 37 & 1.637 \\
\hline Embarazos clínicos & 459 & 73 & 112 & 8 & 652 \\
\hline Partos & 308 & 54 & 70 & 5 & 437 \\
\hline Recién nacidos & 386 & 69 & 89 & 6 & 550 \\
\hline
\end{tabular}

la transferencia de al menos cuatro embriones. $\mathrm{O}$ en ciclos de OD, cuando la calidad de los ovocitos es óptima, se transfiere tres embriones en el $14 \%$ de los casos. Esta política de transferencia de embriones se traduce, lamentablemente, en una estabilización en la frecuencia de partos múltiples en los ciclos de FIV/ICSI, es así que poco más de un quinto de los partos son múltiples. Aunque afortunadamente, los partos triples han desaparecido.

La frecuencia de complicaciones de los procedimientos de reproducción asistida es baja. De hecho sólo se registraron cinco casos de hiperestimulación ovárica y uno de hemorragia durante la culdocentesis, lo que corresponde a un riesgo de 0,5\% de los ciclos iniciados. Esto puede corresponder a un subregistro, sin embargo es alentador tanto para las pacientes como para sus médicos tratantes.

\section{CONCLUSIÓN}

Esta versión del Registro Chileno de Reproducción Asistida muestra un aumento en el número de ciclos registrados, aunque el acceso en nuestro país está todavía muy lejos de los países desarrollados. Persiste la alta frecuencia de fecundación por ICSI, llegando a representar el $89 \%$ de los ciclos iniciados en el 2011. La tasa de parto de los ciclos de IVF/ ICSI se mantiene relativamente estable en relación a años anteriores. Gracias a la criopreservación y la transferencia secuencial de toda la cohorte de embriones producidos, la frecuencia de partos triple descendió considerablemente. Sin embargo, la alta frecuencia de parto doble no ha experimentado ninguna disminución y debe ser el próximo desafío. El resumen de la experiencia en 2011 se presenta en la Tabla VI.

Agradecimientos: El presente registro corresponde a la información otorgada voluntariamente por las siguientes centros de medicina reproductiva: Centro de Estudios Reproductivos, Clínica de la Mujer y Medicina Reproductiva, Clínica Sanatorio Alemán, Instituto de Investigación Materno Infantil, UMR Clínica Alemana, UMR Clínica Las Condes, UMR Clínica Monteblanco.

\section{REFERENCIAS}

1. Zegers-Hochschild F, Nygren KG, Adamson GD, de Mouzon J, Lancaster P, Mansour R, et al. The International Committee Monitoring Assisted Reproductive Technologies (ICMART) glossary on ART terminology. Fertil Steril 2006;86(1):16-9.

2. Ferraretti AP, Goossens V, de Mouzon J, Bhattacharya S, Castilla JA, Korsak V, et al. Assisted reproductive technology in Europe, 2008: results generated from European registers by ESHRE. Hum Reprod 2012;27(9):2571-84. 